\title{
Single Step Channeling in Glass Interior by Femtosecond Laser
}

\author{
Panjawat Kongsuwan, Hongliang Wang, Y. Lawrence Yao \\ Department of Mechanical Engineering \\ Columbia University \\ New York, NY, USA
}

\begin{abstract}
Channeling inside a transparent material, glass, by femtosecond laser was performed by using a single step process rather than hybrid processes that combine the laser irradiation with an additional tool or step to remove the material. Tightly focusing of a single femtosecond laser pulse using proper optical and laser processing parameters could induce the microexplosion and could create voids inside transparent materials, and the effects of these parameters on the resultant feature geometry and channel length were studied. Understanding of the channel length variation at different locations from the specimen surface could enhance prediction capability. Taking into account of the laser, material, and lens properties, numerical models were developed to predict the absorption volume shape and size at different focusing depths below the surface of a specimen. These models will also be validated with the variation in feature and channel lengths inside the specimen obtained from the experiments. Spacing between adjacent laser pulses and laser parameters were varied to investigate effects of channel overlapping and its influence on long channel formation.
\end{abstract}

\section{INTRODUCTION}

Microchannels are the essential features in micro-fluidic devices, micro-total analysis systems ( $\mu$-TAS), and lab-ona-chip (LOC) devices for biomedical applications. Lab-ona-chip devices are microsystems integrated with functional components such as micro-optics, waveguides and microfluidics aiming at the miniaturization onto a single substrate of several functionalities. LOCs use networks of microfluidic channels to transport, mix, separate, react and analyze very small volumes of biological samples. Several substrate materials including silicon, glass and polymers are used for LOC fabrication. However, glass is still the material of choice for many applications due to its chemical inert, stable in time, hydrophilic, nonporous, optically clear, and easily supports electro-osmotic flow [1]. Currently, fabrication of microfluidic devices still heavily relies on photolithographic techniques, which require multilayer and multistep processing procedures to form 3D microstructures.

Femtosecond laser micromachining has emerged as a revolutionary technique for creating 3D microfluidic structures inside transparent substrates [2]. Laser irradiation inside transparent materials using the fluence equal or above the material damage threshold can alter the material structure and resulting mainly in positive/negative refractive index change. For higher fluence, a strong lasermatter interaction is occurred, and micro-explosion confined in the focal volume takes place with creation of voids. Glezer et al. [3] tightly focused femtosecond laser pulses to initiate micro-explosions inside transparent materials and found that submicrometer structures or voxels can be produced inside the materials. Juodkazis et al. [4] showed that the nanovoid in glass is formed as a result of shock and rarefaction waves at pulse power much lower than the threshold of self-focusing. Gamaly et al. [5] determined the mechanism of void formation as a result of micro-explosion and analyzed the size of the void as a function of the deposited energy. Using this mechanism, there is potential to form 3D patterns of voids inside glass.

Currently, there are mainly two strategies for fabricating 3D microchannels embedded in glass using femtosecond laser. The first strategy employs femtosecond laser direct writing followed by chemical etching in either silica glass or photosensitive glass. Hnatovsky et al. [6] used the combination of femtosecond laser dielectric modification and selective chemical etching to fabricate microchannels in fused silica and BK7 borosilicate glass, and also investigated the optimum irradiation conditions needed to produce high-aspect ratio microchannels with small symmetric cross-sections and smooth walls. Sun et al. [7] demonstrated the dependence of the microchannels fabricated in fused silica glass using chemical etching on the femtosecond lasers pulses with different central wavelengths. Wang et al. [8] formed the 3D microfluidic structures by irradiating femtosecond laser into a photosensitive Foturan glass, thermal annealing to produce crystallites of lithium metasilicates in the laser-irradiated regions, and selectively chemical etching those regions in a diluted hydrofluoric acid solution. Another strategy is to perform femtosecond laser drilling from the rear surface of the glass in contact with distilled water, by which the water introduced into the microchannel can help remove the ablated material [2]. Hwang et al. [9] fabricated microfluidic channel in fused silica by water-assisted femtosecond drilling, and clarified that the debris is removed with the help of bubbles generated in the channel. Iga et al. [10] investigated how the diameters of the channels drilled from the rear surface of silica glass by a 
femtosecond laser depend on the incident energy and the number of laser pulses. An et al. [11] machined combined structures consisting of cascaded microchambers and microtrenches inside silica glass by water-assisted ablation with femtosecond laser pulses. The first process is the limited by the etch ratio between the irradiated and unirradiated areas, and it usually get the taper channels. For the second process, the ablated debris can no longer be ejected from the microchannel when the length of the channel increases.

In this study, rather than using hybrid processes such as liquid assisted femtosecond laser processing or femtosecond laser irradiation combined with subsequently chemical etching the treated region, single-step channeling is investigated by attempting to connect the voids created from each single femtosecond laser pulse irradiation. This novel process uses single femtosecond laser pulses instead of pulse trains at a specific repetition rate, and does not require an additional process to remove material from the channel. In order to understand the channel formation mechanism, to get the acceptable channel geometry, and to develop prediction capability, the effects of optical and laser parameters on the resultant morphology by single femtosecond laser pulse are studied. Numerical models are developed to predict the feature shape and lengths which also correspond to the channel lengths. The possibility of using the numerical features length simulated by absorption volume modeling and electromagnetic diffraction modeling as a guideline to estimate the experimental results is also discussed.

\section{BACKGROUND}

\section{Channel formation and focusing effect}

When a femtosecond laser pulse with high energy in the order of microjules is tightly focused into fused silica, nonlinear absorption will occur, a high number density of free electrons will be generated inside the absorption volume due to multi-photon and avalanche ionization. These hot free electrons will transfer their energy to ions, and thermal equilibrium of free electrons and lattice will be attained in picoseconds. In addition to a thermal process in which phase transitions take place, high pressure within the absorption volume builds up. When a high pressure and temperature volume is created inside this restricted volume, there is hydrodynamic expansion which proceeds as a micro-explosion. Shock wave emerges from this volume, compressing the surrounding material, and simultaneously rarefaction wave behind the shock front propagates in the opposite direction creating a void $[12,13]$.

Spherical aberration caused by focusing through the airsample refractive index mismatched interface determined the variation of the threshold pulse energy as a function of the focusing depth and the size of the modified zones in fused silica [14]. Marcinkevicius et al. [15] reported that the spherical aberrations increased the size and distorted the shape of the photo-damaged region. Liu et al. [16] experimentally studied the influence of the focusing depth on both the index change threshold and damage threshold as well as on the cross section of the fabricated waveguides under irradiation of $1-\mathrm{kHz}$ femtosecond laser pulses.

When the laser beam is focused through the air-glass interface, the paraxial focus or the focus produced by the central area of a focusing lens is located at the distance which is the product of the refractive index of air and the focusing depth $\left(n_{2} d\right)$. By paraxial approximation and using the Snell's law on the interface, $n_{2} \sin \theta_{2}=n_{1} \sin \theta_{1}=N A$ is satisfied, and the longitudinal aberration range, $l_{p a}$, due to spherical aberration, which is the distance from the paraxial-ray focal plane to the peripheral-ray focal plane can be written as $[16,17]$

$$
l_{p a}=\frac{\tan \theta_{1}}{\tan \theta_{2}} d-n_{2} d=\left[\sqrt{\frac{n_{2}^{2}-N A^{2}}{1-N A^{2}}}-n_{2}\right] d
$$

where $\theta_{1}$ is incident angle of focusing beam, $\theta_{2}$ is the refracted angle of the focusing beam, $n 2$ is the refractive index of glass, $d$ is the focusing depth from the interface, and NA is the numerical aperture of an objective lens. On the other hand, by using diffraction theory and taking into account the variation in size of the converging Gaussian beam, $w(z)=w_{0}\left[1+\left(\theta z / w_{0}\right)^{2}\right]^{1 / 2}$ is satisfied. The diffraction limited spot size location, $f$, will be related to the focusing depth, the converging incident angle, and the converging refracted angle as : $f=\theta_{1} d / \theta_{2}$, and longitudinal aberration range, $l_{d f}$, due to spherical aberration, which is the distance from the diffraction limited focal plane to the peripheral-ray focal plane can be written as

$$
l_{d f}=\frac{\tan \theta_{1}}{\tan \theta_{2}} d-\frac{\theta_{1} d}{\theta_{2}}=\left[\sqrt{\frac{n_{2}^{2}-N A^{2}}{1-N A^{2}}}-\frac{\sin ^{-1}(N A)}{\sin ^{-1}\left(N A / n_{2}\right)}\right] d
$$

Either the longitudinal aberration range in Eq.(1) or that in Eq.(2) will have an influence on the nonlinear energy deposition by spreading the intensity distribution along its range. Therefore, the feature or the channel resulting from the laser-matter interaction region due to nonlinear absorption inside transparent materials will be proportional to the focusing depth.

\section{Numerical analysis}

By taking into account the effect of the longitudinal aberration range, the numerical model based on the electromagnetic diffraction of a laser beam focused through an air-glass interface could be constructed to predict the absorption volume shape and size at different focusing depths below the surface of a specimen. A laser beam of known power arrives at the interface through the first medium of refractive index $n_{1}$, and the interface at the surface of a specimen separates the medium of refractive index $n_{1}$ from the second medium of refractive index $n_{2}$. The electromagnetic field before the interface can be expressed as a superposition integral that sums up all 
possible plane waves propagating within the convergence angle of the high-aperture lens. Each plane wave is transmitted through the interface obeying the Fresnel refraction law. Using the fields calculated in the first medium as boundary conditions to obtain solution for the diffraction problem using a second integral representation of the electromagnetic field in the second medium. The resulting electric field distribution in the second medium is then a solution of the time-independent wave equation and Maxwell's equations and can be written as $[18,19]$

$$
\begin{gathered}
E_{2 x}(x, y, z)=\frac{-i k_{1} f l_{0}}{2}\left[I_{0}+I_{2} \cos \left(2 \theta_{p}\right)\right] \\
E_{2 y}(x, y, z)=\frac{-i k_{1} f l_{0}}{2} I_{2} \sin \left(2 \theta_{p}\right) \\
E_{2 z}(x, y, z)=\frac{-i k_{1} f l_{0}}{2} I_{1} \cos \theta_{p}
\end{gathered}
$$

where $k_{1}$ is wave number in the first medium, $f$ is the focusing distance, $l_{0}$ is the field amplitude factor, $x$ and $y$ are the positions in lateral directions, $z$ is the position in axial direction, and $\theta_{p}=\tan -1(y / x)$ is the inclination angle. The integrals $I_{0}, I_{1}$, and $I_{2}$ are given by [18]

$$
\begin{aligned}
I_{0}= & \int_{0}^{\alpha} \sqrt{\cos \phi_{1}} \sin \phi_{1} \exp \left[-i d\left(k_{1} \cos \phi_{1}-k_{2} \cos \phi_{2}\right)\right] \\
& \times\left(\tau_{s}+\tau_{p} \cos \phi_{2}\right) J_{0}\left(k_{1} \sqrt{x^{2}+y^{2}} \sin \phi_{1}\right) \\
& \times \exp \left(i k_{2} z \cos \phi_{2}\right) d \phi_{1} \\
I_{1}= & \int_{0}^{\alpha} \sqrt{\cos \phi_{1}} \sin \phi_{1} \exp \left[-i d\left(k_{1} \cos \phi_{1}-k_{2} \cos \phi_{2}\right)\right] \\
& \times \tau_{p} \sin \phi_{2} J_{1}\left(k_{1} \sqrt{x^{2}+y^{2}} \sin \phi_{1}\right) \\
& \times \exp \left(i k_{2} z \cos \phi_{2}\right) d \phi_{1} \\
I_{2}= & \int_{0}^{\alpha} \sqrt{\cos \phi_{1}} \sin \phi_{1} \exp \left[-i d\left(k_{1} \cos \phi_{1}-k_{2} \cos \phi_{2}\right)\right] \\
& \times\left(\tau_{s}-\tau_{p} \cos \phi_{2}\right) J_{2}\left(k_{1} \sqrt{x^{2}+y^{2}} \sin \phi_{1}\right) \\
& \times \exp \left(i k_{2} z \cos \phi_{2}\right) d \phi_{1}
\end{aligned}
$$

where $\alpha$ is the angular semi-aperture of the lens, $k_{2}$ is wave number in the second medium, $\phi_{1}$ is the incident angle on the interface, $\phi_{2}$ is the refracted angle from the interface, $\tau_{s}$ and $\tau_{p}$ are the Fresnel coefficients, and $J_{0}, J_{1}$, and $J_{2}$ are the Bessel function of the first kind and order zero, one, and two, respectively. Hence the distribution of the timeaveraged electric energy density, $w_{e}$, and the total energy density, $w$, inside the specimen near a focal plane are [19]

$$
\begin{aligned}
\left\langle w_{e}(x, y, z)\right\rangle= & \frac{k_{1}^{2} f^{2} l_{0}^{2}}{64 \pi}\left\{\left|I_{0}\right|^{2}+4\left|I_{1}\right|^{2} \cos ^{2} \theta_{p}+\left|I_{2}\right|^{2}\right. \\
& \left.+2 \cos \left(2 \theta_{p}\right) \operatorname{Re}\left(I_{0} I_{2}^{*}\right)\right\} \\
\langle w(x, y, z)\rangle & =\frac{k_{1}^{2} f^{2} l_{0}^{2}}{32 \pi}\left\{\left|I_{0}\right|^{2}+2\left|I_{1}\right|^{2}+\left|I_{2}\right|^{2}\right\}
\end{aligned}
$$

where $*$ is the conjugate of an complex number. The electric energy density and total energy density of points in the vicinity of a focal plane will be significantly high, and when compared to the material damage threshold, the absorption volume could be identify to predict the size of features and channels at different focusing depths below the top surface of transparent materials.

\section{EXPERIMENTAL SETUP}

Single step channeling is achieved by using a chirped amplified Ti:Sapphire laser system which outputs high energy ultrashort pulses with $800 \mathrm{~nm}$ wavelength and 130 fs pulse duration at a $1 \mathrm{kHz}$ repetition rate. The commercial grade S1-UV fused silica of $3.0 \mathrm{~mm}$ thick was cut to approximately $6 \mathrm{~mm} \times 12 \mathrm{~mm}$ size. The laser beam was focused by $40 \mathrm{x}$ objective lens with NA 0.6 inside the specimen. Different conditions of laser processing and optical parameters were applied by varying the energy of the laser pulses and focusing depth with respect to the top surface of the material. Long channels were obtained by cascading each channel generated by single laser pulses along an optical axis. Different laser pulse energy and focusing depth conditions were used to investigate the size of features and channels and to investigate the effects of channel overlapping and its influence on long channel formation.

After the femtosecond laser irradiation, transmission light differential interference contrast (DIC) optical microscopy was used to obtain the cross section view (xzplane) of the features and channels. The samples were ground and polished with lapping films to the line across channels. Refractive DIC optical microscopy and AFM topography were used to verify the existence of cavities on the polished surface. Raman spectroscopy was also carried out to further support whether the channels are real cavities.

\section{RESULTS AND DISCUSSION}

\section{Channel geometry}

In order to achieve long micro-scale channels in glass materials, other studies have used hybrid processes such as the combination of ultrashort laser irradiation and chemical etching or the liquid-assisted drilling by ultrashort laser scanning along an optical axis. However, single femtosecond laser pulse irradiation using high laser pulse energy and high numerical aperture of objective lens can also produce the reasonable long channels. Figure 1 shows the axial cross section (xz-plane) of features and channels created inside fused silica sample using laser pulse energy of $10 \mu \mathrm{J}, 20 \mu \mathrm{J}$, and $30 \mu \mathrm{J}$, respectively at the same focusing depth of $1500 \mu \mathrm{m}$ below the top surface of $3 \mathrm{~mm}$ thick fused silica with standard flatness of 3-5 waves/inch from transmission DIC optical microscopy. The features defined by the distinctive color change around irradiated 


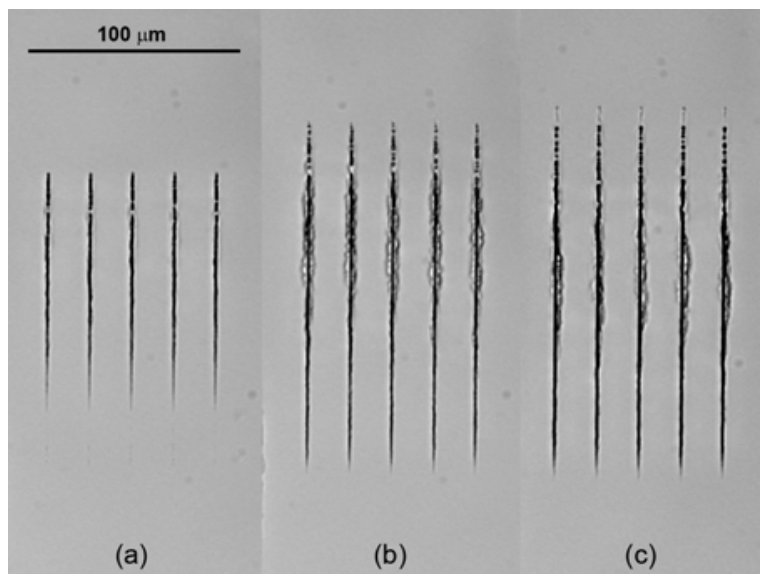

Figure 1. Transmission DIC optical microscopy of cross section view (xz-planes) of features and channels at same focusing depth of $1500 \mu \mathrm{m}$ using pulse energy of (a) $10 \mu \mathrm{J}$, (b) $20 \mu \mathrm{J}$, and (c) $30 \mu \mathrm{J}$. The above three figures have been adjusted to the same scale.

regions due to material alteration in optical and mechanical properties, have high aspect ratios (length/width). The feature is strongly dependent on laser pulse energy as shown in Fig. 1, and the formation of larger features is corresponded to the higher the laser pulse energies. Inside the features, there are dark core regions, and these regions could be the consequence of the high level of interaction which subsequently generates high temperature and high pressure, and eventually causes the material explosion inside laser focal volume. The dark color regions are believed to be cavities, and the longest uniform dark color area inside the feature is identified as a channel from single laser pulse irradiation. The features and channels have a long and narrow tear drop shape corresponding to the shape of absorption volume.

To verify whether the identified channel is really a cavity, the fused silica sample was ground and polished from the top surface until the new polished surface was located at a line across channels. Figure 2 shows the radial

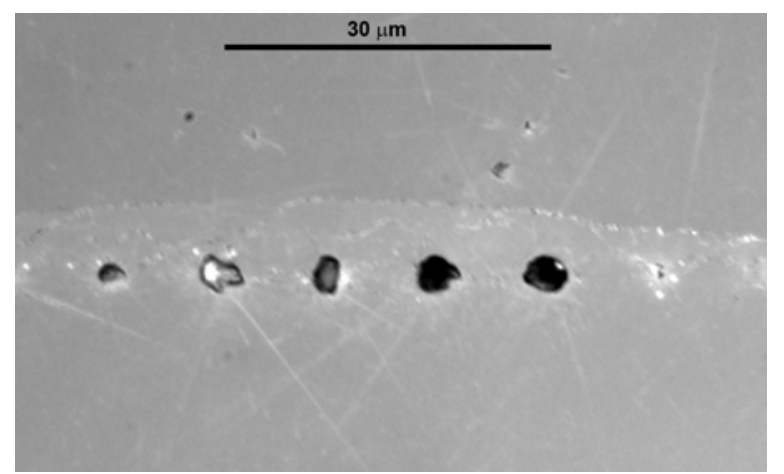

Figure 2. Refractive DIC optical microscopy of top view (xy-plane) of the new polished surface across the channels irradiated by a series of single pulse energy of $30 \mu \mathrm{J}$. cross section (xy-plane) of the polished sample irradiated by a series of single pulse with pulse energy of $30 \mu \mathrm{J}$ from reflective DIC optical microscopy. From the optical reflective image in Fig. 2, the cross section of channels looks dark as well as the optical transmitted image, and the radial cross section shape of features and channels is oval or almost circular shape. The size of features and channels in this $x-y$ view image is about a few micrometers corresponding well with the $\mathrm{x}-\mathrm{z}$ view image. The cavity on the surface of transparent material normally looks dark when observed with the reflective optical microscope; therefore, these identified channels could be assumed as cavities as well. However, the Atomic Force Microscopy (AFM) measurements of topography of channels in radial cross section were also performed to assure that the channels are cavities. The 3D topographic image of four distinctive channels with $10 \mu \mathrm{m}$ spacing is illustrated in Fig. 3(a). The AFM top view image and line profiles across channels can also be seen in Fig. 3(b), and from the vertical line profile it can be concluded that the channel size in the direction perpendicular to the laser beam is about a few micrometers for laser pulse energy of $30 \mu \mathrm{J}$. In the range of this study, the widths of microchannels may be limited to a few microns in size, however, in manufacturing, a much bigger size could be achieve if using significantly high power femtosecond laser. In addition to the optical microscopy and AFM topography images, the Raman spectroscopy was also used to measure structural changes at and around the feature and channel inside the fused silica sample. Raman signal measurements were performed in a series of fifteen points along the line cross the representative channel in $x-z$ view as illustrated in Fig. 4(a). Raman spectral analysis of fused silica reveals that there are three-, four-, and five- and six-fold rings structure in the material, and these rings structure correspond to the 606 $\mathrm{cm}^{-1}, 495 \mathrm{~cm}^{-1}$, and $440 \mathrm{~cm}^{-1}$ peak in Raman spectra, respectively. The lowest peak intensity of all ring structures

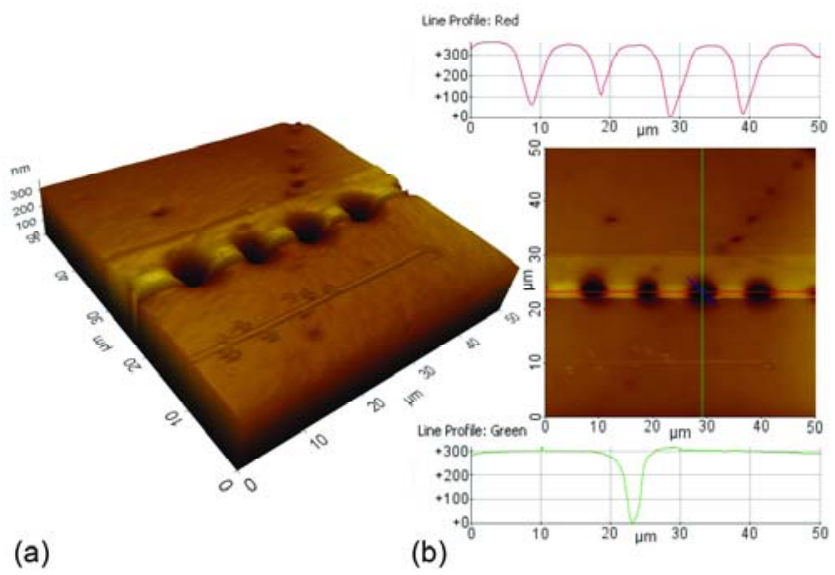

Figure 3. (a) 3D AFM topography and (b) the AFM top view image and line profiles across the channels on the new polished surface (xy-plane) across the channels irradiated by a series of single pulse energy of $30 \mu \mathrm{J}$. 

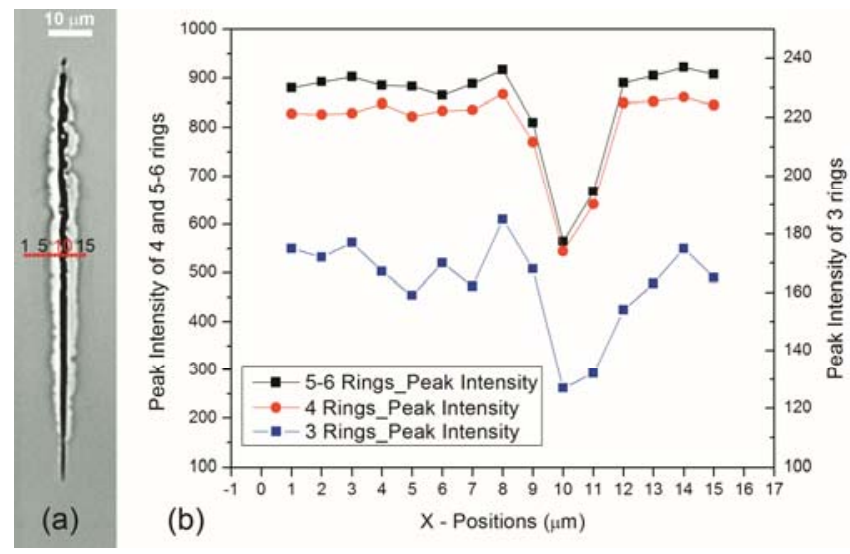

Figure 4. (a) Raman signal measurements points across the cross section view (xz-plane) of a feature and channel, and (b) the peak intensity of the 3 rings, 4 rings, and 5-6 rings structure of fifteen points across the channel.

at point 10 which locates at center of the representative channel, and lower peak intensity at its adjacent points compared to other points in the reference region as plotted in Fig. 4(b) reply that there are low detected volumes of material in the vicinity of those points. This Raman results could be indirectly verified that the identified channels are cavities as well. Therefore, the optical microscopy, AFM topography, and Raman spectroscopy support each other that the defined channels are cavities.

Figure 5 shows the shape and size of features and channels created inside fused silica sample using same laser pulse energy of $30 \mu \mathrm{J}$ at different focusing depth in the range of $500-2500 \mu \mathrm{m}$ below the top surface within $3 \mathrm{~mm}$ thick of the material from the $x-z$ view. It can be seen that the size especially in the optical direction (z-axis) is strongly

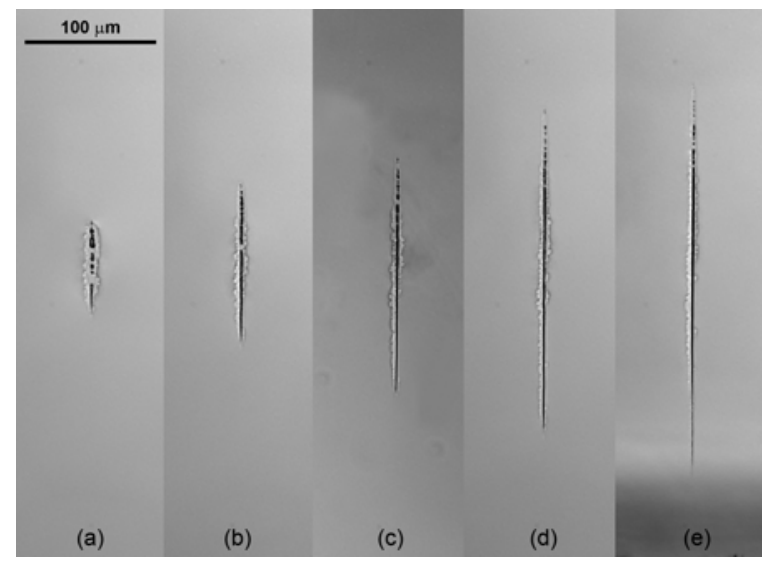

Figure 5. Transmission DIC optical microscopy of cross section view (xz-planes) of features and channels using same pulse energy of $30 \mu \mathrm{J}$ at different focusing depths of (a) $500 \mu \mathrm{m}$, (b) $1000 \mu \mathrm{m}$, (c) $1500 \mu \mathrm{m}$, (d) $2000 \mu \mathrm{m}$, and (e) $3000 \mu \mathrm{m}$. The above five figures have been adjusted to the same scale. dependent on the focusing depth. The greater the focusing depths correspond to the generation of longer features and channels. The variation in size in the optical direction is caused by spherical aberration due to the focusing of laser beam through the refractive index mismatched air-glass interface. On one hand, the plane of focal volume will move to the lower location than the Gaussian focus plane (if there is no interface) due to the refraction of laser beam. On the other hand due to the presence of the air-glass interface, the focused rays inside laser beam cone, which initially converge to the single focal plane at different angle, will diversely propagate to different focal planes in glass, and this phenomenon results in a longitudinal aberration range. The deeper the focusing depths will correspond to the occurrence of longer aberration ranges.

Figure 6 shows the feature lengths at different focusing depths of pulse energies of $10 \mu \mathrm{J}, 20 \mu \mathrm{J}$, and $30 \mu \mathrm{J}$, respectively, compared to longitudinal aberration ranges in Eq.(1) defined as Paraxial aberration ranges in background section. The feature lengths have increasing trends with the focusing depths with respect to the top surface of samples, and range from 23 to 283 microns in length and 4 to 46 in aspect ratio. As seen in Fig. 6, the feature lengths depend on both focusing depths and laser pulse energies. The greater the focusing depth, the longer the feature length; the length of the feature also increases with laser pulse energy. The features lengths of all pulse energies in this study fall within the paraxial aberration ranges. This could be due to the deposited laser pulse energy will be absorbed and broadly distributed in this paraxial aberration ranges, and the absorbed energy density of points only within this ranges will probably be greater than the material damage threshold. It results in feature lengths shorter than the paraxial aberration ranges. The feature lengths show leveling trends at deep focusing depths, and due to the spreading of lower energy within the same aberration ranges

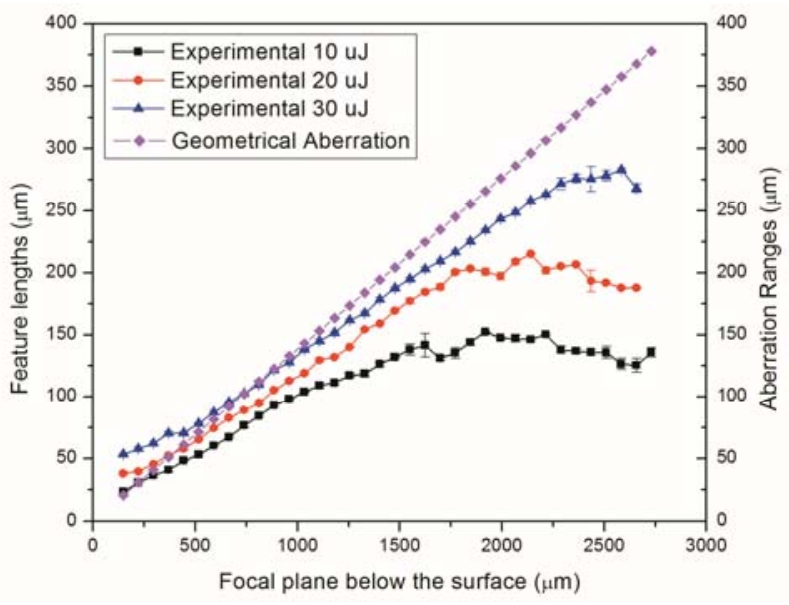

Figure 6. Comparison of experimental feature lengths at 10 $\mu \mathrm{J}, 20 \mu \mathrm{J}$, and $30 \mu \mathrm{J}$, and the paraxial aberration range at different focusing depths. Error bars denote standard deviation. 
caused by the lower absorbed energy density, the lower pulse energies level off at shallower focusing depths compared to the higher ones as observed in Fig. 6 . Although the paraxial aberration ranges are not perfectly matched the feature lengths, a guideline that the feature lengths will be approximately equal or less than the paraxial aberration ranges could be used.

Figure 7 shows the channel lengths at different focusing depths of pulse energies of $10 \mu \mathrm{J}, 20 \mu \mathrm{J}$, and $30 \mu \mathrm{J}$, respectively, compared to longitudinal aberration ranges in Eq.(2) defined as diffraction limited aberration ranges in background section. In the range of this study with respect to focusing depths, the channel lengths have only an increasing trend for the case of $30 \mu \mathrm{J}$ pulse energy, but also show leveling and decreasing trends for the case of $10 \mu \mathrm{J}$ pulse energy. It replies that at further depth locations the pulse energy will be over spreading such that the absorbed energy density that is greater than the material damage threshold occurs in shorter and shorter ranges, and the material explosion will happen in shorter ranges as well resulting in shorter channel lengths as clearly seen in case of $10 \mu \mathrm{J}$ in Fig. 7. The diffraction limited aberration ranges are sometimes shorter or longer than the feature lengths at different laser pulse energies in this study, however, they are quite close to and always greater than the channel lengths of all pulse energies and focusing depths. Therefore, quick guideline could also be used to estimate that the resulting channel lengths will be less than the diffraction limited aberration ranges.

By cascading a single pulse channel along the optical axis within the thickness of fused silica sample using a long working distance objective lens, the connection of channels can be performed to produce a long channel which has adequate length for most microfluidic and lab-on-a-chip applications. However, the appropriate overlapping distance of single channel has to be considered. Figure 8(a) and 8(b)

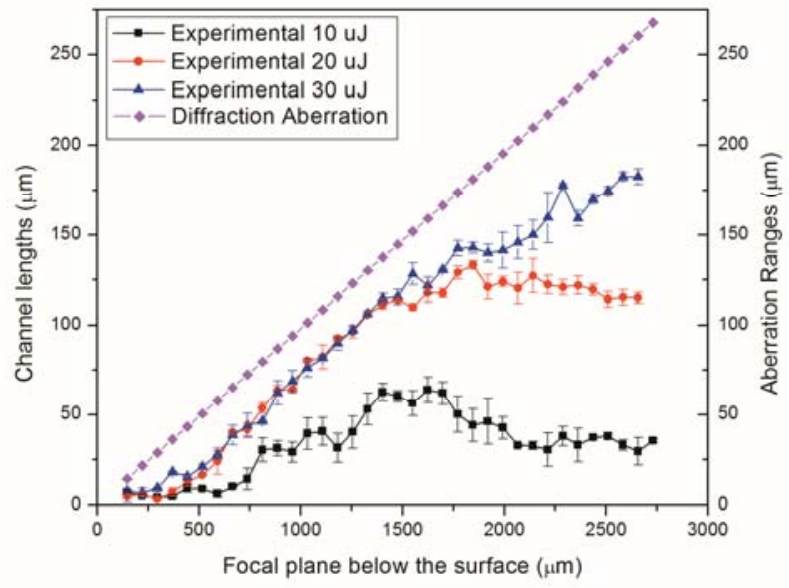

Figure 7. Comparison of experimental channel lengths at $10 \mu \mathrm{J}, 20 \mu \mathrm{J}$, and $30 \mu \mathrm{J}$, and the diffraction limited aberration range at different focusing depths. Error bars denote standard deviation.

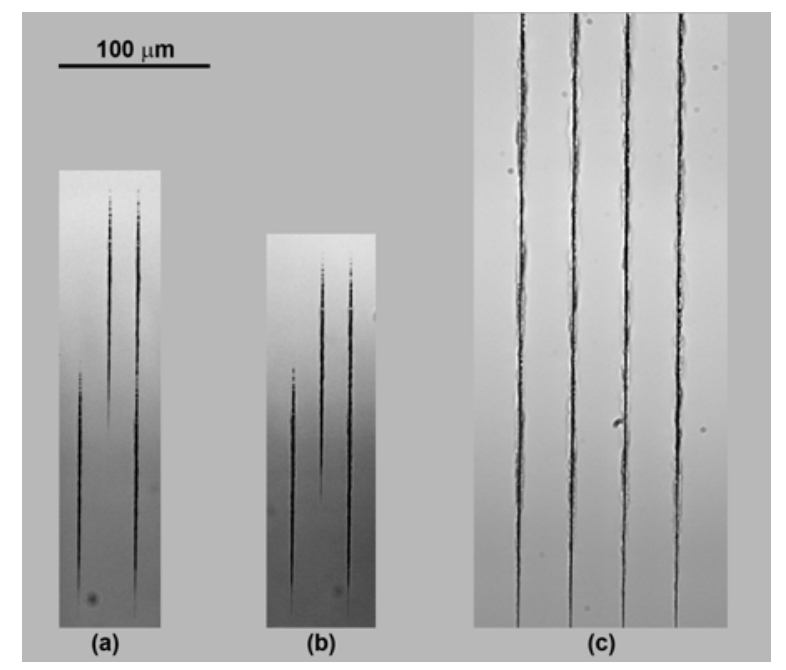

Figure 8. Cascading of two single pulses with (a) an inadequate overlapping distance, and (b) an appropriate overlapping distance. (c) Cascading of multiple single pulses using pulse energy of $30 \mu \mathrm{J}$. The above three figures have been adjusted to the same scale.

show the transmission optical DIC image in xz-plane of cascading channels using different overlapping distance for two single pulses. The left and the middle one are the locations of the bottom and the top channels that will be cascading, respectively. The right channels are the results of cascaded channels. As seen in Fig. 8(a), the two channels are not completely connected due to an inadequate overlapping distance. When the overlapping distance is increased, the cascaded channel has uniform dark color in the overlapping region, which implies that the channel cascading can be succeeded if an appropriate overlapping distance has been used. Figure 8(c) shows the representative cascaded channels of multiple single pulses. From observation by optical microscope, the channel cascading is usually succeeded if the overlapping distance of the features is equal to or greater than $68 \%$. Although the microchannels by single-step channeling process can be performed only in the direction that is paralleled to the incident laser beam and the shape of microchannels has to be simple and straight rather than contour or complex, this novel process could still be a potential alternative to produce micro-scale channels inside transparent materials for microfluidic and lab-on-a-chip applications. For its industrial applications, setups during processing may be required if there are changes in directions of microchannels, and the connecting shapes at different angles or the effects of non-perpendicular surface with respect to the incident laser beam on the shape and size of microchannels could be further explored.

\section{Numerical Modeling}

From experimental results, the shape and size of features and channels varies as a function of focusing depth. 
In order to predict the shape and size of those features and channels at different focusing depths below the specimen surface, the two numerical models of laser absorption volume and electric energy density field inside a transparent material were constructed. The first numerical model considers the temporal profile and spatial intensity of Gaussian laser beam which propagates through a focusing lens and an air-glass interface to points in the vicinity of a focal plane, and determines breakdown points which have intensity greater than the material intensity damage threshold. The second numerical model considers the electric field inside the second medium after focusing electromagnetic wave through a focusing lens and an interface between two different mediums, then determines the electric energy density field in the vicinity of a focal plane, and compares it with the energy density damage threshold of the material. Both numerical models will take effect of the longitudinal aberration ranges into account.

Absorption volume modeling. From temporal and spatial distribution characteristics of femtosecond laser pulses, the intensity of an unfocused laser beam in each time slice was determined. When the unfocused laser beam has been converged to the smaller area in the vicinity of a focal plane, the magnified intensity will be compared to the material intensity damage threshold, and the breakdown points which have intensity greater than the threshold will be determined [20]. Consequently, the corresponding locations in the optical axis of these breakdown points will be calculated considering focusing parameters, optical properties of two materials, and aberration effects from airglass interface. Eventually, all breakdown points will combine and generate the absorption volume. Figure 9 shows the representative simulated axial cross section (rzplane) of the absorption volume by $30 \mu \mathrm{J}$ pulse energy at the focusing depth of $1000 \mu \mathrm{m}$ below the top surface of fused silica material. The cross section shape of the absorption volume looks like the teardrop shape and has high aspect ratios (length/width). It is interesting that the top breakdown point in optical axis of this representative absorption volume is occurred closely to the diffraction limited focal plane at $-1040.4 \mu \mathrm{m}$ rather than the paraxial focal plane at $-1000 \mu \mathrm{m}$. The size of the absorption volume is indeed dependent on the focusing depths and laser pulse energies, and the longest distance along the optical axis of the absorption volume is defined as the numerical feature lengths simulated by the absorption volume modeling. More details of the results from this numerical model will be shown and verified with the other numerical model and the experimental results in next section.

Electromagnetic diffraction modeling. A superposition of plane waves inside mediums can represent electromagnetic fields. Taking into account the optical properties of two different mediums, the focusing parameters, and Fresnel's refraction law at the interface

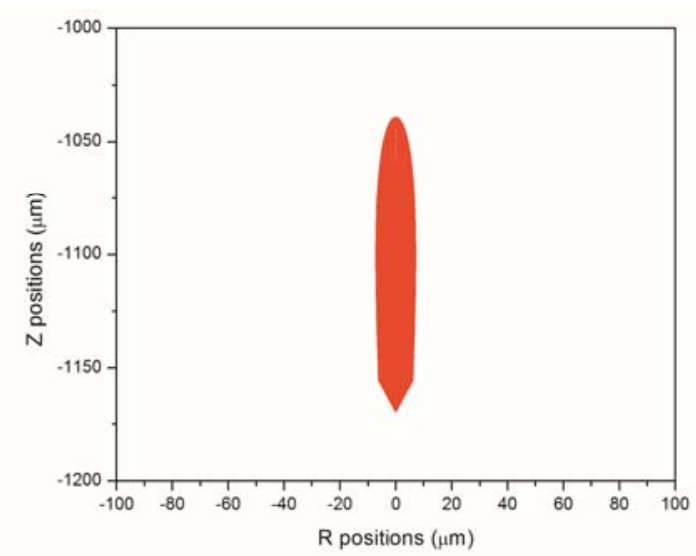

Figure 9. The representative simulated cross section (rzplane) of the absorption volume by $30 \mu \mathrm{J}$ pulse energy at the focusing depth of $1000 \mu \mathrm{m}$ from the absorption volume model.

between two different mediums, a superposition of refracted plane waves can construct the time-independent or time-averaged electric and magnetic fields. The area in the vicinity of the focal plane which has high enough electric energy density especially greater than the damage threshold of the material can be counted as a material structural alteration area which represents the feature and channel generated by a single femtosecond laser pulse. Figure 10 illustrates the 3D surface map of representative electric energy density field on the xz-plane along an optical axis in the vicinity of the focal plane for laser pulse energy of 30 $\mu \mathrm{J}$ at the focusing depth of $1000 \mu \mathrm{m}$ below the top surface of fused silica material. The region of interest is located between the focal plane of axial and peripheral rays within the angular semi-aperture of the objective lens with numerical aperture (NA) equal to 0.6. As seen in Fig. 10, the

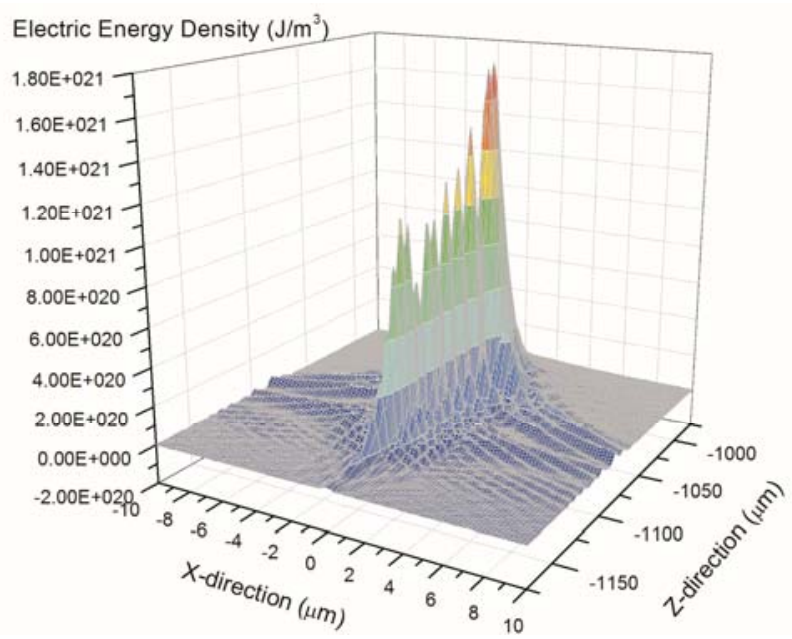

Figure 10. The 3D surface map of representative electric energy density field on the xz-plane along an optical axis in the vicinity of the focal plane for laser pulse energy of 30 $\mu \mathrm{J}$ at the focusing depth of $1000 \mu \mathrm{m}$. 


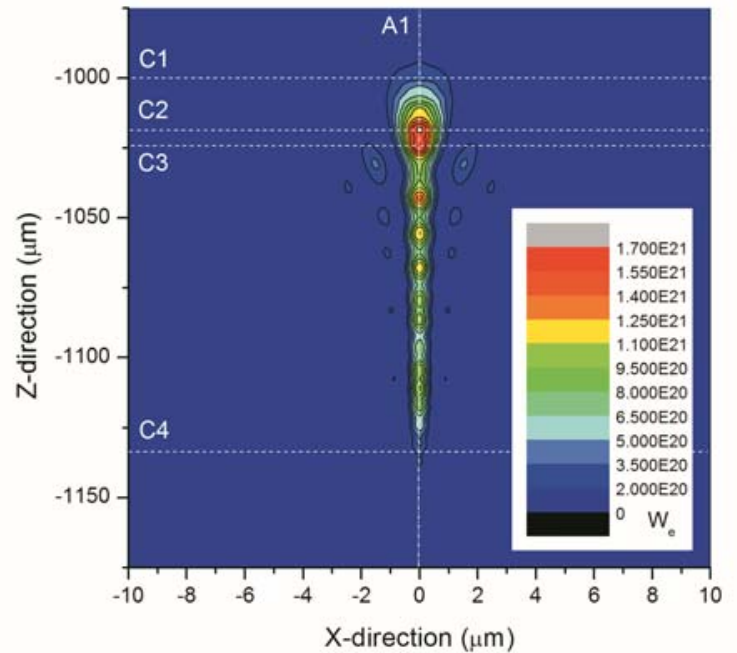

Figure 11. The contour map of representative electric energy density field on the xz-plane along an optical axis in the vicinity of the focal plane for laser pulse energy of 30 $\mu \mathrm{J}$ at the focusing depth of $1000 \mu \mathrm{m}$. Lines C1, C2, C3, C4, and $\mathrm{A} 1$ represent section lines across this field.

electric energy density is extremely high only near the optical axis between the paraxial focal plane at $-1000 \mu \mathrm{m}$ and the peripheral focal plane at $-1138.4 \mu \mathrm{m}$ below the top surface of the material.

The contour map of electric energy density in this region of interest by $30 \mu \mathrm{J}$ pulse energy at $1000 \mu \mathrm{m}$ focusing depth is shown in Fig. 11. This Contour looks like tear drop shape and similar to the appearance of features and channels, and the total length and width of this contour are also about the same size as the experimental features in
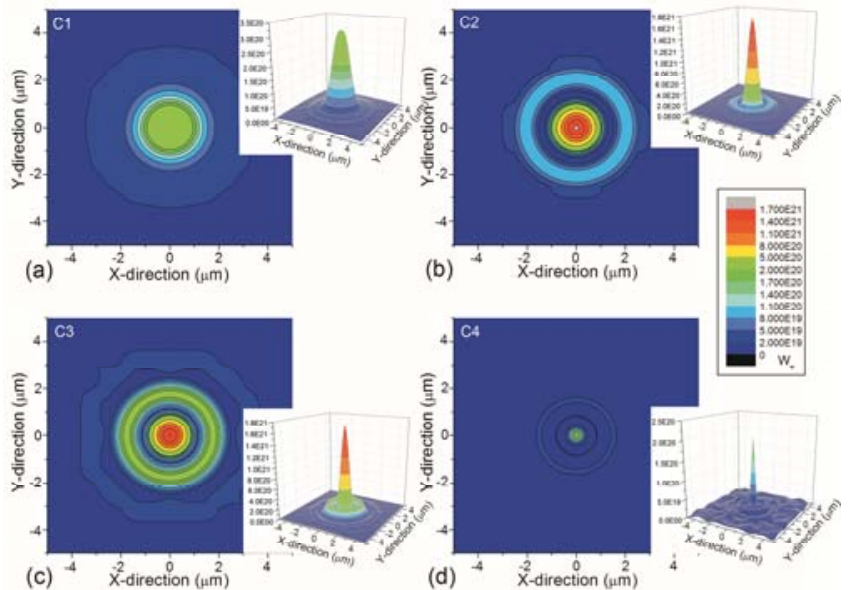

(c) ${ }^{-4} x^{-2}$-direction $\left(\mu m^{2}\right)$

Figure 12. The contour and 3D surface maps in xy-planes along (a) line $\mathrm{C} 1$ located along the paraxial-ray focal plane, (b) line $\mathrm{C} 2$ located along the highest peak of electric energy density, (c) line C3 located along the second highest peak of electric energy density, and (d) line C3 located along the peripheral-ray focal plane.

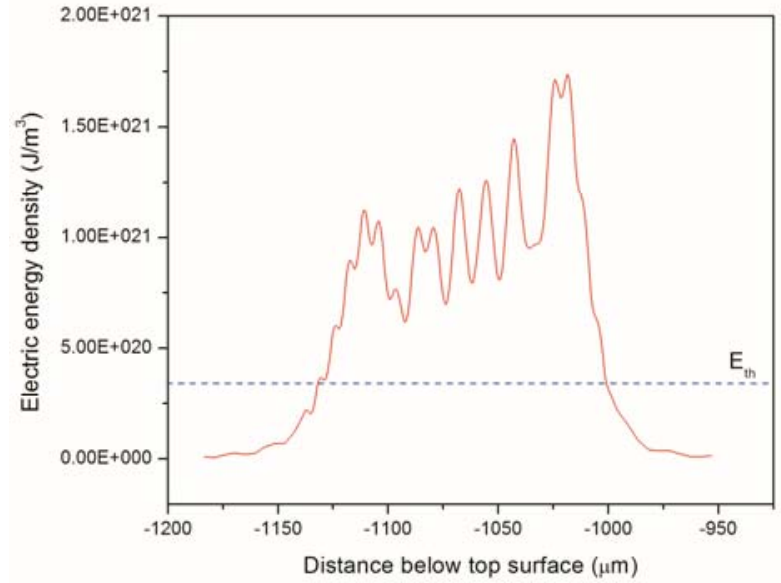

Figure 13. The electric energy density on the optical axis along line A1 in Fig. 11 compared to the damage threshold of fused silica sample.

Fig. 5(b). In the Fig. 11, lines C1, C2, C3, C4, and A1 represent section lines across this representative high electric energy density field. Lines $\mathrm{C} 1$ and $\mathrm{C} 4$ are located along the paraxial- and peripheral-ray focal planes, respectively. Lines $\mathrm{C} 2$ and $\mathrm{C} 3$ are located along the top two peaks of electric energy density, and line A1 is located along the optical axis. The contour and 3D surface maps on xy-planes along lines $\mathrm{C} 1, \mathrm{C} 2, \mathrm{C} 3$, and $\mathrm{C} 4$ are illustrated in Fig. 12(a), (b), (c), and (d), respectively. From these contour and 3D surface maps, the distribution of electric energy density along axes perpendicular to the laser propagation direction has a Gaussian profile. The peak energy density of electric field on both paraxial- and peripheral-ray focal planes in Fig. 12(a) and (d) are slightly less than the material damage threshold, and this could give the answer why the experimental feature lengths in previous section are usually shorter than the defined paraxial aberration. The contour maps in Fig. 12(b) and (c) along the xy-planes which have top two peaks of electric energy density show multiple rings which have energy density greater than the material damage threshold. The size of outbound rings with such high electric energy density is approximately $2 \mu \mathrm{m}$, and it can be seen that this size is corresponded to the width of experimental features and channels in previous section. Figure 13 shows the electric energy density on the optical axis along line A1 in Fig. 11. The oscillation of the electric energy density along this optical axis could be due to the constructive and destructive interference of phase factor resulting from a superposition of refracted plane waves. From the line profile of electric energy density along the optical axis, the numerical feature length simulated by the electromagnetic diffraction model is defined as the distance whereas the electric energy density is greater than the material damage threshold $\left(\mathrm{E}_{\mathrm{th}}\right)$ as illustrated in Fig. 13. The numerical feature lengths from this model are strongly dependent on both the focusing depths and the laser pulse energies. More details of the results from this numerical 
model will be shown and verified with the previous numerical model and the experimental results in next section.

Validation of numerical models. From the simulated absorption volumes similar to the representative cross section map in Fig. 11, and the simulated electric energy density line profiles along the optical axis similar to the representative profile in Fig. 14, the numerical feature lengths calculated by absorption volume and electromagnetic diffraction models at different focusing depths below the top surface of fused silica sample using the single femtosecond laser pulse energy of $30 \mu \mathrm{J}$ as well as the experimental feature lengths are plotted in Fig. 14. The feature lengths from both numerical models have increasing trends with the focusing depths, and line up pretty well with the experimental feature length results. Especially for this particular laser pulse energy of $30 \mu \mathrm{J}$, the results from the electromagnetic diffraction model almost perfectly match the experimental results and are better predict the feature lengths than the absorption volume modeling. Figure 15 shows the numerical and experimental feature length as a function of laser pulse energy at two different focusing depths of $1000 \mu \mathrm{m}$ and $1500 \mu \mathrm{m}$ below the top surface of fused silica sample, respectively. As seen in Fig. 15, the electromagnetic diffraction model has some difficulties in determining the feature length when the laser pulse energy is less than $20 \mu \mathrm{J}$ due to the electric energy density become lower than the material damage threshold at low pulse energies, and the level of laser pulse energy at which feature lengths could not be defined is also dependent on the focusing depths. The deeper the focusing depths, the greater the spreading of electric energy density resulting in the higher levels of pulse energy at which the feature lengths could not be determined. This problem could be due to the time-independent or time-averaged based approximation of the model whereas the absorption

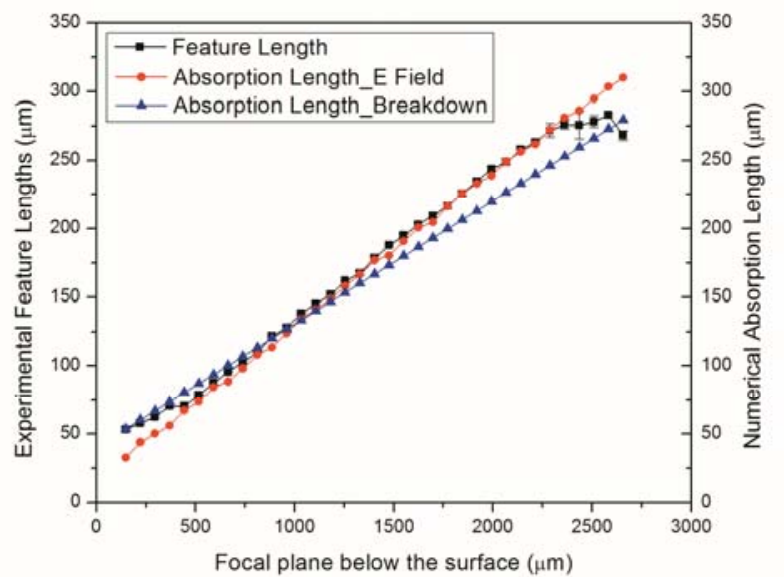

Figure 14. Comparison of feature lengths from two numerical models and experimental results at different focusing depths using laser pulse energy of $30 \mu \mathrm{J}$.

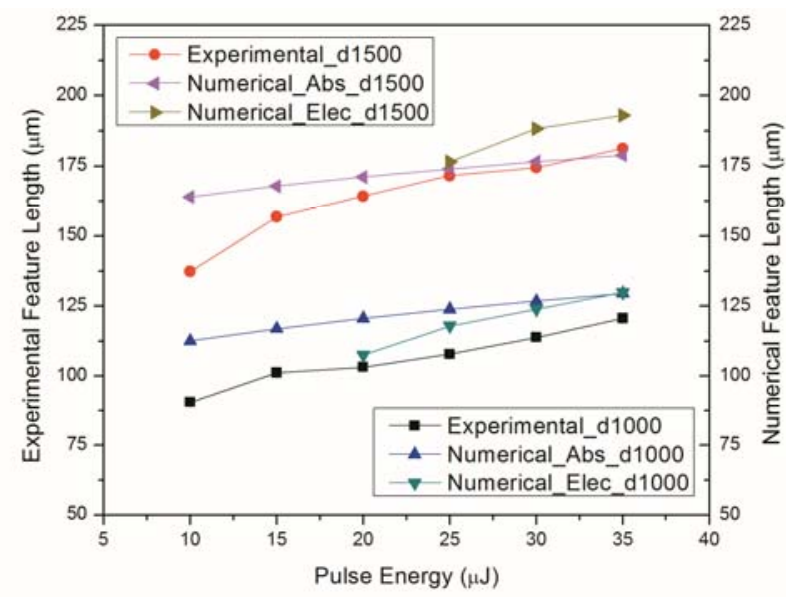

Figure 15. Comparison of feature lengths from two numerical models and experimental results at different pulse energies for focusing depth of $1000 \mu \mathrm{m}$ and $1500 \mu \mathrm{m}$ below the top surface of fused silica sample, respectively.

volume model which considers the temporal profile of laser pulse as well as the spatial profile still be able to predict the feature lengths at low pulse energy, however, the discrepancy between the numerical and experimental results will increase correspond to the lower pulse energies. Therefore, depending on the working parameters, the electromagnetic diffraction model better predicts the feature lengths with some restrictions at low pulse energies.

\section{CONCLUSION}

Single step channeling inside fused silica glass was performed by a series of single femtosecond laser pulses. The axial cross section of the generated features by the transmission DIC optical microscopy revealed that they range from 23 to 283 microns in length and 4 to 46 in aspect ratio. Those features also have a long uniform dark colored region, which is identified as a microchannel. The radial cross sectioning of this channel in conjunction with the surface topography confirmed that the channels are cavities. The reflective optical DIC microscopy and Raman spectroscopy also support this fact. The size of the features and channels is strongly dependent on the laser pulse energies and focusing depths. The variation in size of features and channels with focusing depths is due to aberration caused by the refractive index mismatch at airglass interface. With overlapping distance of the feature equal or greater than $68 \%$, channel cascading could be successfully performed to produce a longer channel in millimeter scale. Two numerical models were developed to investigate the shape and size of features and channels generated by single femtosecond laser pulses at different laser pulse energies and focusing depths. The numerical results were validated using the experimental ones, and both absorption volume and electromagnetic diffraction models could be used to estimate the feature lengths. 


\section{ACKNOWLEDGMENT}

This work is partially supported under NSF grant number: CMMI-0936171. Financial support from the Royal Thai government is also gratefully acknowledged. Research carried out in part at the Center for Functional Nanomaterials, Brookhaven National Laboratory, which is supported by the U.S. Department of Energy, Office of Basic Energy Sciences, under Contract No. DE-AC0298CH10886.

\section{REFERENCES}

[1] Osellame R., Hoekstra H. J. W. M., Cerullo G., and Pollnau M., 2011, "Femtosecond laser microstructuring: an enabling tool for optofluidic labon-chips," 463(3), pp. 442-463.

[2] Liao Y., Ju Y., Zhang L., He F., Zhang Q., Shen Y., Chen D., Cheng Y., Xu Z., Sugioka K., and Midorikawa K., 2010, "Three-dimensional microfluidic channel with arbitrary length and configuration fabricated inside glass by femtosecond laser direct writing.," Optics letters, 35(19), pp. 32257.

[3] Glezer E. N., and Mazur E., 1997, "Ultrafast-laser driven micro-explosions in transparent materials," Applied Physics Letters, 71(7), p. 882.

[4] Juodkazis S., Misawa H., Hashimoto T., Gamaly E. G., and Luther-Davies B., 2006, "Laser-induced microexplosion confined in a bulk of silica: Formation of nanovoids," Applied Physics Letters, 88(20), p. 201909.

[5] Gamaly E., Juodkazis S., Misawa H., Lutherdavies B., Rode A., Hallo L., Nicolai P., and Tikhonchuk V., 2008, "Formation of nano-voids in transparent dielectrics by femtosecond lasers," Current Applied Physics, 8(3-4), pp. 412-415.

[6] Hnatovsky C., Taylor R. S., Simova E., Rajeev P. P., Rayner D. M., Bhardwaj V. R., and Corkum P. B., 2006, "Fabrication of microchannels in glass using focused femtosecond laser radiation and selective chemical etching," Applied Physics A, 84(1-2), pp. 4761.

[7] Sun Q., Saliminia A., Th F., and Chin S. L., 2008, "Microchannel fabrication in silica glass by femtosecond laser pulses with different central wavelengths," 18, pp. 3-6.

[8] Wang Z., and Zheng H., 2009, "Ultrashort laser subsurface micromachining of three - dimensional microfluidic structures inside photosensitive glass," Laser and Particle Beams, pp. 521-528.

[9] Hwang D. J., Choi T. Y., and Grigoropoulos C. P., 2004, "Liquid-assisted femtosecond laser drilling of straight and three-dimensional microchannels in glass," Applied Physics A: Materials Science \& Processing, 79(3), pp. 605-612.
[10] Iga Y., Ishizuka T., Watanabe W., Itoh K., Li Y., and Nishii J., 2004, "Characterization of Micro-Channels Fabricated by In-Water Ablation of Femtosecond Laser Pulses," Japanese Journal of Applied Physics, 43(No. 7A), pp. 4207-4211.

[11] An R., Li Y., Dou Y., Liu D., Yang H., and Gong Q., 2006, "Water-assisted drilling of microfluidic chambers inside silica glass with femtosecond laser pulses," Applied Physics A, 83(1), pp. 27-29.

[12] Gamaly E. G., Luther-Davies B., Hallo L., Nicolai P., and Tikhonchuk V. T., 2006, "Laser-matter interaction in the bulk of a transparent solid: Confined microexplosion and void formation," Physical Review B, 73(21), pp. 214101-1-15.

[13] Hallo L., Mézel C., Bourgeade A., Hébert D., Gamaly E. G., and Juodkazis S., 2010, "Laser-Matter Interaction in Transparent Materials : Confined Microexplosion and Jet Formation," Extreme Photonics \& Applications, T.J. Hall, S.V. Gaponenko, and S.A. Paredes, eds., Springer Netherlands, pp. 121-146.

[14] Hnatovsky C., Taylor R. S., Simova E., Bhardwaj V. R., Rayner D. M., and Corkum P. B., 2005, "Highresolution study of photoinduced modification in fused silica produced by a tightly focused femtosecond laser beam in the presence of aberrations," Journal of Applied Physics, 98(1), p. 013517.

[15] Marcinkevičius a, Mizeikis V., Juodkazis S., Matsuo S., and Misawa H., 2003, "Effect of refractive indexmismatch on laser microfabrication in silica glass," Applied Physics A: Materials Science \& Processing, 76(2), pp. 257-260.

[16] Liu D., Li Y., An R., Dou Y., Yang H., and Gong Q., 2006, "Influence of focusing depth on the microfabrication of waveguides inside silica glass by femtosecond laser direct writing," Applied Physics A, 84(3), pp. 257-260.

[17] Sun Q., Jiang H., Liu Y., Zhou Y., Yang H., and Gong Q., 2005, "Effect of spherical aberration on the propagation of a tightly focused femtosecond laser pulse inside fused silica," Journal of Optics A: Pure and Applied Optics, 7(11), pp. 655-659.

[18] Török P., Varga P., Laczik Z., and Booker G. R., 1995, "Electromagnetic diffraction of light focused through a planar interface between materials of mismatched refractive indices: an integral representation," Journal of the Optical Society of America A, 12(2), p. 325.

[19] Richards B., and Wolf E., 1959, "Electromagnetic Diffraction in Optical Systems. II. Structure of the Image Field in an Aplanatic System," Proceedings of the Royal Society A: Mathematical, Physical and Engineering Sciences, 253(1274), pp. 358-379.

[20] Kongsuwan P., and Yao Y. L., 2010, "Transmission Welding of Glasses by Femtosecond Laser: Structural and Mechanical Properties," Proc. 29th International Congress on Applications on Lasers \& Electro-Optics, Anaheim, CA, pp. 1069-1078. 